\title{
Evaluating the Implementation of a Pragmatic Person-Centered Communication Tool in the Nursing Home via the RE-AIM Framework.
}

Katherine Abbott ( $\square$ abbottkm@miamioh.edu )

Miami University https://orcid.org/0000-0002-6815-2065

\section{Alexandra Heppner}

Miami University

Nytasia Hicks

Miami University

Abigail Hermesch

Miami University

Kimberly VanHaitsma

Pennsylvania State University School of Nursing: The Pennsylvania State University College of Nursing

\section{Research}

Keywords: Nursing Home, Implementation, evidence-based, person-centered care, preferences, communication

Posted Date: October 9th, 2020

DOI: https://doi.org/10.21203/rs.3.rs-86502/v1

License: (9) (1) This work is licensed under a Creative Commons Attribution 4.0 International License. Read Full License 


\section{Abstract}

Background: Person-centered care (PCC) is a philosophy that recognizes "knowing the person" and honoring individual preferences. The purpose of this study was to evaluate the implementation of a novel PCC communication tool in nursing homes $(\mathrm{NH})$. The tool is based on an assessment of $\mathrm{NH}$ resident likes and dislikes via the Preferences for Everyday Living Inventory (PELI), which is an evidenced-based, validated instrument used to enhance the delivery of PCC. The Preferences for Activity and Leisure (PAL) Card was developed to communicate residents' preferences for leisure and activities across care team members.

Methods: Providers were recruited to utilize the recreation and leisure items from the PELI to assess resident important preferences and create PAL Cards for 15-20 residents. Providers collected data aligned with the RE-AIM framework (Reach, Effectiveness, Adoption, Implementation, Maintenance).

Results: Reach and Adoption: A total of $n=43$ providers registered and $n=26(60 \%)$ providers completed the project. Ownership of participating providers included $54 \%$ not for profit, $46 \%$ for profit, and $8 \%$ government owned with an average star rating of 3.7 (SD 1.1) and a range of 1-5. Effectiveness and Implementation: Participants attempted $n=424$ PAL Cards and completed $n=406$. The average acceptability of the intervention measure (AIM) was 4.7 (SD 0.4), intervention appropriateness measure (IAM) was 4.5 (SD 0.5), and feasibility of intervention measure (FIM) was 4.6 (SD 0.5). Maintenance: Providers were able to complete $82 \%$ of PAL Card placement over the course of 5 months with few cards going missing $(<2 \%)$.

Conclusion: The majority of providers were successful in implementing PAL Cards for residents and reported the intervention as highly acceptable, appropriate, and feasible. The pragmatic PAL Card intervention can assist nursing home providers in meeting PCC regulations and contribute to building relationships between residents, family, and staff.

\section{Contributions To The Literature}

- The Preferences for Activity and Leisure (PAL) Card was developed to communicate nursing home residents' preferences for leisure and activities across care team members.

- Ohio providers, coached virtually by an external advisor, completed over 400 PAL Cards with fidelity to the protocol and found them to be acceptable, feasible, and appropriate.

- Discussions with the external advisor helped providers adapt the intervention to their local contexts while facilitating information sharing with other providers.

- PAL Cards are inexpensive, flexible to staff time limitations, and adds to the capacity of organizations to deliver valued person-centered outcomes.

\section{Background}


Nursing homes $(\mathrm{NH})$ are shifting toward a person-centered philosophy of care (PCC). PCC seeks to understand each individual's preferences, goals and values, and to honor them throughout the care delivery process [1-2]. The Centers for Medicare and Medicaid Services (CMS), the largest payer of longterm services and supports (LTSS), has supported PCC via federally mandated regulations. For example, all CMS funded NHs are required to assess a subset of individual-level preferences annually through the Minimum Data Set (MDS 3.0) Preference Assessment Tool [3]. The goal for assessing important preferences is that they can guide day-to-day care leading to better outcomes for residents. Interventions that tailor care to individual needs and preferences lead to improved positive affect [4], greater engagement, alertness, and attention [5], decreases in hallucinations, agitation, anxiety, aggression, sleep disorders, and aberrant motor behavior [6]. However, communicating resident preferences across employees in different departments and shifts has proved difficult to implement in the dynamic, complex care environment of the nursing home [7].

Person-centered tools that communicate individuals' psychosocial needs have been tested in both acute and long-term care settings, with demonstrated positive outcomes on communication and care. In hospitals, interventions including patient whiteboards [8] and the Tell-Us Card [9] improved the quality of care and increased patients' involvement and knowledge of their care. Evidence-based communication tools in nursing homes include using Talking Mats [10] and memory books [11] that are used to improve communication for individuals with dementia. Collecting and sharing residents' life stories is another common strategy to communicate biographical information[12]. Evidence-based interventions such as these continue to play an important role in enhancing the person-centeredness of healthcare organizations.

One type of implementation science is quality improvement (QI), which is done routinely in NHs. CMS requires $\mathrm{NHs}$ to participate in quality assurance and performance improvement activities (QAPI). However, NH providers struggle to engage and sustain QAPI because of resource constraints, staff turnover, and heavy workloads [13]. To successfully and sustainably engage nursing home providers in adopting evidence-based practices, implementation science principles are needed [14]. Therefore, the purpose of this study was to evaluate the implementation of an evidence-based communication intervention call Preferences for Activity and Leisure (PAL) Cards.

Intervention Co-Development

$\mathrm{NH}$ providers explained that after assessing an individual's preferences, they struggled to communicate the information across care team members. Providers asked for a quick way to reference individual resident preferences that could travel with them throughout the organization, such as on the back of their wheelchair, or attached to a walker. We collaborated with four nursing home providers to co-design a communication tool called PAL Cards based upon evidence-based communication models used in similar settings $[8,11]$. PAL Cards are $5 \times 7$ inch, portable cards that display a resident's name, biography, and important activity preferences, such as hobbies (see Fig. 1). We utilized the Readiness Assessment 
for Pragmatic Trials (RAPT) framework to help guide the development of the intervention protocol to ensure that the intervention was designed pragmatically [15].

\section{Preference for Activity and Leisure (PAL) Card Intervention Protocol}

Step 1: Selecting preference items. The intervention protocol starts with helping providers select items that would allow them to better assess an individual resident's important preferences. Providers were given the choice to either use the 8 activity items from the Preference Assessment Tool (PAT) in the Minimum Data Set (MDS) 3.0 [16] or select additional items from the Preferences for Everyday Living Inventory (PELI) PELI-NH [17]. The PELI-NH includes 72-questions that cover five psychosocial preference domains including (1) Self-Dominion (e.g., having privacy, choosing what to eat), (2) Enlisting others in care (e.g., having a caregiver be of the same gender), (3) Leisure and Diversionary (e.g., watching television), (4) Social Contact (e.g., meeting new people), and (5) Growth Activities (e.g., exercise). Respondents are asked to respond to a question stem that starts with "How important is it to you to... [insert preference]" with a 4-point Likert scale of 1 (very important) to 4 (not important at all) and have additional prompts that allow providers to delve more deeply into the specific aspects of resident preferences. The PELI-NH is a tool that has been subject to extensive reliability and validity development for pragmatic use in the nursing home setting [17-19] and heavily informed the development of the PAT.

Because the PELI-NH items have been developed to be ideographic, "stand alone" individual items (PELI$\mathrm{NH}$ does not have traditional nomothetic scaled scores), providers had the option of selecting which and how many items they wished to use in their assessment process. At a minimum, providers were encouraged to use the 8 activity items from the PAT since those items are routinely captured in required assessment processes in all nursing homes that receive CMS reimbursement for clinical services, making these items the ideal pragmatic starting point for any provider. The 33 recreation and leisure items from the PELI-NH were the maximum number of items providers selected from (and included the 8 PAT items). Providing access to the wider array of PELI-NH recreational preference items allowed maximum flexibility and options for customizing to individual community goals for honoring resident preferences.

Step 2: Interviewing the resident. While conducting PELI interviews, we suggested that staff take note of any preferences that the resident reports as particularly important, as these details are incorporated into the resident's PAL Card.

Step 3: Creating the PAL Card. After the interview is completed, providers download the PAL Card template from a freely available website. For ease of use, the template form is a Word document with fixed fields allowing direct entry of information gathered during the interview. The front of each PAL Card includes space for a short biography. The back of the card is personalized to highlight six recreation and leisure preference items that were reported as particularly significant or "important" during their interview. The sections on the back will vary depending on the unique interests of each resident (See Fig. 1). 
Step 4: Consultation with resident and/or family member to verify accuracy. After a PAL Card is created it is recommended that the information is reviewed with the resident and or family proxy to ensure it accurately reflects his/her interests before it is laminated. If the resident or proxy notes any discrepancies, the card is updated with the correct information by a staff member.

Step 5: Placement of PAL Card. Once the PAL Card is reviewed for accuracy, the resident is asked where they would like the card to be placed. We recommend that it be placed on an assistive mobility device (e.g., wheelchair or walker) so that the card can travel with the individual throughout the community. Alternatively, the PAL Card can also be adhered to a resident's door. Some providers may choose to place the cards in one central location, such as a break room bulletin board.

Ohio nursing homes are required to complete one quality improvement project (QIP) approved by the Ohio Department of Aging (ODA) every other year [20]. To facilitate alignment of the intervention with stakeholder priorities to comply with this requirement, the PAL card intervention protocol was designed to be utilized in the context of a QIP to enhance communication of important resident leisure preferences in their daily life.

\section{Methods}

Aim, Design, and Setting: We sought to evaluate the implementation of the PAL Card QIP with NH providers participating in a six-month quality improvement project who were trained and coached monthly. We utilized the RE-AIM framework [21] to evaluate the QIP (see measures below for more details). The Standards for Reporting Implementation Studies (STARi) checklist was utilized [22].

Recruitment strategies. The PAL Card QIP was advertised for two months on the ODA website [23] and included in a monthly newsletter disseminated to all nursing home administrators in Ohio $(n=960)$. In addition, we discussed the project during a presentation at an industry organized state level activities professional conference to approximately 80 individuals. Providers were asked to complete a short online questionnaire or contact the first author to sign up for the project. Any $\mathrm{NH}$ provider within and outside the State of Ohio was eligible to participate.

Implementation strategy. We conducted an initial one-hour virtual training for all participants via Webex. Per best practice recommendations from the Institute for Healthcare Improvement (IHI), we encouraged providers to start small and pilot the PAL Card intervention with approximately $15-20$ residents. Starting with a pilot allows for the collection of information needed to either decide whether or not they have the necessary resources to implement the intervention with all residents in their community [24]. The initial training also included guidance on all the steps in the intervention protocol described above. Participants received a tour of PreferenceBasedLiving.com, where all materials needed for the project (PELI Interviews, editable PAL Card Word Document template, and PAL Card Implementation tip sheet with step-by-step instructions) were made available at no cost. 
The project manager created a checklist that clearly delineated each step of the process, data collection forms that identified what data to record, and instructions for joining WebEx meetings. Once participants began the project, we responded to requests for creation of additional resources such as a tip sheet for obtaining background information and a sample PAL Card policy. Following the initial training session, we utilized a monthly virtual group-coaching model to replicate a virtual learning circle collaborative [2526]. The goal of the monthly coaching calls was to give providers the opportunity to problem solve common solutions together. We utilized Sign-up Genius to schedule conference calls allowing providers to sign up for days/times that were convenient for them each month.

Project requirements for successful completion. Criterion used to define successful completion of the program were developed in coordination with ODA. In order for providers to receive a certificate of completion, providers were required to: participate in online training (1 hour), conduct preference interviews (choice of the 8 activity items from MDS 3.0 Section $F$ or selection of up to 33 items from PELI$\mathrm{NH}$ ), participate in monthly virtual coaching calls, orient staff to PAL Cards, create and place PAL Cards for 15-20 residents, collect data, submit pictures of placed PAL Cards, and complete a final individual telephone interview. The ODA also required providers to document in residents' care plans their consent to having a PAL Card made and displayed publicly.

\section{MEASURES}

This study utilized Reach, Effectiveness, Adoption, Implementation, and Measurement (RE-AIM) to evaluate the PAL Card intervention [21]. The measurement of each RE-AIM element was as follows: Reach was assessed by the number of providers who responded out of the total number of providers invited. Potential participants in this QIP study included all $980 \mathrm{NHs}$ in the state of Ohio. Reach was calculated by the number of $\mathrm{NHs}$ out of 980 that indicated an interest in participating in the QIP program. Effectiveness was assessed by staff ratings of acceptability, feasibility and appropriateness of the PAL Card intervention protocol. We utilized three pragmatic implementation outcomes measures, 1) Acceptability of Intervention Measure (AIM), 2) Intervention Appropriate Measure (IAM), and 3) Feasibility of Intervention Measure (FIM) [27]. Acceptability is the perception among stakeholders that the intervention is agreeable, palatable, and satisfactory. Appropriateness is the perceived fit, relevance, or compatibility of the innovation for a given setting, provider, or consumer. Feasibility is the extent to which the intervention can be successfully carried out within the given setting. Each measure consists of 4 items and were asked during the final telephone interview at the conclusion of the project. We inserted the words "PAL Cards" where the scale suggests listing the Evidence-Based Practice (EBP) (e.g., I welcome Pal Cards, The PAL Cards seem fitting, The PAL Cards seem easy to use). Response options include: completely disagree (score $=1$ ), disagree (score=2), neither agree nor disagree (score=3), agree (score=4), and completely agree (score $=5$ ). Higher average scores indicate greater acceptability, appropriateness, and feasibility. The reported Chronbach's alpha coefficients for each measure are high with AIM alpha .84, IAM alpha of .92, and FIM of .92 [27]. 
Adoption was measured by the number of participants who completed all project requirements (e.g., participate in online training, complete PAL Cards with 15-20 residents, complete a final individual phone interview). Implementation was measured by the fidelity to the requirements of the project as well as costs of the intervention, based upon staff time needed to implement the intervention. Implementation costs were estimated by multiplying the average time invested in conducting the interview and creating the PAL Card by average wages of staff involved (e.g., social worker, activities, nursing). We estimated a per resident cost of the intervention to the organization by multiplying the average interview time by the average salaries of occupations (including fringe rates) pulled from salary.com to calculate the cost of conducting the interview, creating the PAL Card, and added in the cost of printing one page in color and lamination. Finally, Maintenance was assessed by whether the PAL Card remained in place (e.g., card still in original location vs. card missing) over five months after initiation of the project.

\section{Analysis}

Descriptive statistics were calculated using IBM SPSS v27.

\section{Results}

\section{Reach \& Adoption}

We had a total of $n=43$ nursing home providers sign up for the project. Interestingly, $n=41$ providers were from Ohio, and two of the organizations were located in other states (Pennsylvania, Virginia). Forty providers attended the virtual training and $n=35$ began implementation (See Fig. 2). For our Adoption measure, $\mathrm{n}=26(74 \%)$ providers completed all requirements of the project. Providers attempted an average of $16.4(S D=6.0)$ PAL Cards and completed 15.9 (SD = 6). In total, 424 PAL Cards were attempted and 406 PAL Cards were completed and placed.

The characteristics of providers who completed the project included $54 \%(n=14)$ not for profit or government owned, and $46 \%(n=12)$ that were for profit. Their average bed size was 87 ; while their average star rating was 3.7 (SD 1.1) with a range from 1-5 (See Table 1). The characteristics of providers who did not complete the project included $13 \%(n=2)$ not-for-profit and $87 \%(n=13)$ for profit. Their average bed size was 72 while their average star rating was 3.4 (SD 0.7) with a range from $1-5$ (see Table 1). 
Table 1

Characteristics of Organizations who Completed and Did Not Complete the PAL

Card Project.

\begin{tabular}{|lll|}
\hline & Completers $(\mathbf{n}=\mathbf{2 6})$ & Non Completers $(\mathbf{n}=\mathbf{1 5})$ \\
\hline Ownership & & \\
Not-For-Profit/Government & $\mathrm{N}=14(54 \%)$ & $\mathrm{N}=2(13 \%)$ \\
For-Profit & $\mathrm{N}=12(46 \%)$ & $\mathrm{N}=13(87 \%)$ \\
\hline Average Bed Size (SD) & $87(42.0)$ & $74(8.0)$ \\
\hline Average Star Rating (SD) & $3.73(1.1)$ & $3.35(0.7)$ \\
\hline
\end{tabular}

The project manager held 10 training sessions with a total of 40 participants (an average of 4 participants per training and a range of 1-6). In addition, the project manager held a total of 37 monthly coaching calls in the subsequent three months after the initial training (an average of 12 per month). Monthly calls had fewer participants with an average of 2 participants lasting 18 minutes (range 3-35 minutes).

\section{Effectiveness}

Providers reported that the PAL Card intervention was highly acceptable, feasible, and appropriate (see Table 2). The Chronbach's alpha of the measures in this study were 0.79 for the AIM, 0.85 for the FIM, and 0.92 for the IAM.

Table 2

The Acceptability, Feasibility, and Appropriateness of the PAL Card Intervention Measure as Rated by $n=26$ Nursing Home Providers.

\begin{tabular}{|lll|}
\hline & Mean (SD) & Mode \\
\hline Acceptability of Intervention Measure (AIM) & $4.7(0.4)$ & 5 \\
\hline Feasibility of Intervention Measure (FIM) & $4.6(0.5)$ & 5 \\
\hline Intervention Appropriateness Measure (IAM) & $4.5(0.5)$ & 5 \\
\hline
\end{tabular}

\section{Implementation}

Staff from multiple departments contributed to the implementation of $n=424$ PAL cards. Activity professionals completed $72.7 \%$ of PAL cards, volunteers completed $17.3 \%$, nursing $5.7 \%$, and $4 \%$ were completed by another individual (e.g., family, therapy personnel, social worker). Family were engaged in providing some or all information for $11 \%$ of PAL Cards $(n=45)$. Providers were given the choice of which version to use and $40 \%(n=168)$ of PAL Cards were created via the 8 activity items from the MDS Section 
F while $56.4 \%(n=237)$ were created via the 33 -item version. One provider chose to create a version that was 10 items. They selected six items from the MDS activity items (excluded favorite activities and groups of people) and 4 items from the PELI-NH (Reminiscing about the past, playing games, watching or listening to TV, and doing your favorite hobbies).

Providers selecting the 8 activity items from the PAT reported taking an average of 26.8 minutes to complete the interview (SD 20.5 minutes; Median 20 minutes, Mode 30 minutes, Range 5 to 120) and 22.6 minutes to create PAL Cards (SD 19.6 minutes; Median 16 minutes, Mode 10 Minutes, Range 2-120). Providers selecting the 33 activity and leisure items from the PELI-NH reported taking an average of 43 minutes (SD 22.1; Median 40, Mode 30 Minutes, Range 7 to 120 minutes) to complete the interview. For those creating PAL Cards using the 33 items interview it took on average 22 minutes to create PAL Cards (SD 9.5; Median 20, Mode 30, Range 3-50 minutes).

All of the PAL Cards completed $(n=406)$ were reviewed for accuracy by the resident and/or family and were reported to be accurate in $92.5 \%(n=381)$ of cases. For cards that were reported to be inaccurate, individuals wanted to add more details or the family member (e.g., wife, daughter) reviewed the card and found mistakes for individuals who had cognitive impairment. PAL cards were placed on resident's wheelchairs $(n=220 ; 53.5 \%)$, walkers $(n=62 ; 15.1 \%)$, door $(n=93 ; 22.6 \%)$, and other location $(n=36$; $8.8 \%$; e.g., in the resident's room such as the closet door, dresser, over the bed, on the wall, IV pole, gerichair, bulletin board).

Based on the average time to conduct the interview and create the PAL Card, we estimate that implementation costs to the organizations are between $\$ 13.04-\$ 27.35$ per resident. This does not account for time needed for training and depends on whether staff or volunteers are completing the interviews and creating the PAL Cards (see Table 3). In addition, these costs would be in the first three to four months of implementation. It is possible that costs could go down as learning curves flatten. 
Table 3

Estimated Cost of PAL Card Initial Implementation Per Resident.

\begin{tabular}{|lllll|}
\hline Staff Position & $\begin{array}{l}\text { Average Time for Interview and } \\
\text { PAL Card creation in Minutes* }\end{array}$ & $\begin{array}{l}\text { Average } \\
\text { Hourly } \\
\text { Wages }\end{array}$ & $\begin{array}{l}\text { Materials } \\
\text { Cost }\end{array}$ & $\begin{array}{l}\text { Total Cost } \\
\text { of one PAL } \\
\text { Card }\end{array}$ \\
\hline $\begin{array}{l}\text { Activities } \\
(n=292)\end{array}$ & 61 & $\$ 12.68$ & $\$ 0.15$ & $\$ 13.04$ \\
$\begin{array}{l}\text { Volunteer (value of } \\
\text { volunteer time)** } \\
(n=70)\end{array}$ & 55 & $\$ 27.20$ & $\$ 0.15$ & $\$ 27.35$ \\
$\begin{array}{l}\text { Nursing } \\
(n=24)\end{array}$ & 45 & $\$ 27$ & $\$ 0.15$ & $\$ 20.40$ \\
\hline $\begin{array}{l}* \text { Average hourly wages include fringe; **Volunteer hourly wage is presented as the 'value of the } \\
\text { volunteer' [28]. }\end{array}$ & & & & \\
\hline
\end{tabular}

\section{Maintenance}

Across the $\mathrm{n}=26$ providers who completed the project, a total of $n=424$ PAL cards were attempted and (82\%) were completed in five months. Reasons why PAL Cards were not completed included resident refusals $(n=7)$, deaths prior to completion $(n=2)$, discharges prior to completion $(n=1)$, and residents being unable to complete the interview $(n=8)$. The majority of PAL Cards $(68 \%)$ had been placed by month 3 (See Table 4). Once placed, providers tracked whether or not the PAL Card was still in place each month. Providers reported that less than $2 \%$ of the PAL Cards were missing, moved to another location, or needed to be replaced.

Table 4

PAL Card Placement by Month $(n=424)$

\begin{tabular}{|c|c|c|c|c|c|}
\hline & $\begin{array}{l}\text { Month } \\
1\end{array}$ & $\begin{array}{l}\text { Month } \\
2\end{array}$ & $\begin{array}{l}\text { Month } \\
3\end{array}$ & $\begin{array}{l}\text { Month } \\
4\end{array}$ & $\begin{array}{l}\text { Month } \\
5\end{array}$ \\
\hline Not Placed & $63 \%$ & $52 \%$ & $22 \%$ & $9 \%$ & $1 \%$ \\
\hline Placed & $35 \%$ & $43 \%$ & $68 \%$ & $78 \%$ & $82 \%$ \\
\hline $\begin{array}{l}\text { Resident discharged, refused, or passed } \\
\text { away }\end{array}$ & $2.6 \%$ & $4.8 \%$ & $6.7 \%$ & $9 \%$ & $12.5 \%$ \\
\hline Missing & 0 & $0.5 \%$ & $1.5 \%$ & $0.8 \%$ & $1.9 \%$ \\
\hline PAL Card Moved to another location & $\mathrm{n} / \mathrm{a}$ & $\mathrm{n} / \mathrm{a}$ & $0.5 \%$ & $1.8 \%$ & $0.6 \%$ \\
\hline Reprinted and Replaced & $n / a$ & $0.3 \%$ & $0.8 \%$ & $1.5 \%$ & $1.7 \%$ \\
\hline
\end{tabular}




\section{Discussion}

Overall, the majority $(74 \%)$ of providers were successful in completing the PAL Card quality improvement project. Providers implemented PAL Cards with virtual coaching support and reported them to effective in communicating important preferences. The RE-AIM framework was utilized to evaluate the implementation strategy.

Offering the intervention as a state-approved quality improvement project facilitated reaching several providers from across Ohio. By advertising the project through multiple avenues, including conferences and newsletters, providers of varying locations, sizes, for-profit status, and star ratings were reached. Providers dropped out for numerous reasons, but the most common cause was leadership and/or staff turnover. Leadership turnover has been identified as a barrier in other nursing home quality improvement studies [29-30]. Dedicated high-level leaders and project champions are essential to keeping a project on track, so turnover in these key positions made it challenging to move the project forward [13].

Providers who completed the project perceived the intervention to be effective via three pragmatic outcome indicators associated with adoption of evidence-based practices [31]. First, the intervention was reported as acceptable or appealing. Providers welcomed the PAL Cards and reported that they met their approval. Second, the intervention was perceived as appropriate or compatible in the nursing home setting for use with residents and families to communicate important preferences. Providers reported that the PAL cards were suitable and seemed like a good match. Third, PAL Cards were deemed feasible. Providers reported that the evidence-based intervention was implementable, possible, doable, and easy to use. In addition, residents reported that the information on the PAL Cards was highly accurate. This validates prior research that shows that older adults can communicate their preferences, and preferences remain stable over time [32]. These outcomes are relevant to assessing stakeholder's perceptions of interventions, as well as assessing perceptions of implementation strategies. Assessing these outcomes early in the research process may ensure that interventions and implementation strategies are optimized and fit with each user's preferences.

The built-in flexibility of the intervention was an aspect that led to success. Providers had choice and could adapt the intervention to their context in a number of ways. Depending on their perceived bandwidth, providers could choose from a shorter, 8-item interview or a longer 33-item version. Some providers started with 8-items, but added additional items when they felt limited in the information obtained. There was also flexibility in terms of who implemented the intervention. Staff from a variety of positions including social work, activities, nursing, and volunteers took on roles in the project. Some providers used teams of staff, while others took on the project solo (either by choice, or by necessity).

The flexibility in approach led to a wide range of times to complete the interviews and PAL Cards. Some providers viewed the interviews as more of a relationship-building activity, and, for example, interviewed residents over lunch, which increased the time. Other providers took a more focused approached and tried to complete the cards as quickly as possible to fit in with their other job responsibilities. Comfort levels with technology was another factor that influenced the time needed to create the PAL Cards, as some 
providers who lacked the technical skills, such as how to download a file, needed more time to learn the process. The flexibility providers had regarding placement of the PAL Card also assisted with meeting their needs. Our initial recommendation was to have the resident decide where their card was placed, i.e., on their walker, wheelchair, or in their room. During the project, some providers reported it was easier to have a uniform location for all PAL Cards in their community, such as on a bulletin board or by residents' doors. This allowed staff to consistently know where to find the cards. Finally, family members played an important role in the project, especially in providing information about residents' preferences. While we have always acknowledged the potential to involve family in the process, especially as proxies for residents who are unable to communicate, we knew it would add complexity to the project. Therefore, we did not foresee providers involving families to the extent they did in this study, nor did we systematically ask about the assistance from family in the PAL Card process. This is an important consideration for future studies.

The implementation strategy of a virtual coach (one of the research team members) played a vital role in collaborative problem solving with providers. For example, technology was often a barrier and some providers needed one-to-one support with downloading the PAL Card template, printing and laminating the cards, and using video conference software. The coach also helped providers problem solve components of their implementation strategy. Two of the most common conversations were deciding which interview version to use and which residents the provider would trial the project with. Discussions with the coach helped providers adapt the intervention to their local contexts while facilitating information sharing with other providers. Finally, the research team created resources to remediate barriers, when possible. One example was that some providers struggled to collect residents' biographical information needed for the front of the PAL Card. The team created a tip sheet addressing this topic to share with providers. The role of an external advisor as a problem solver has been validated in other nursing home QIPs [13].

We estimated the cost of initial implementation in order to understand the intervention's scalability and impact on resources (e.g., staff time). While the materials cost for the PAL Cards (paper, printing, laminating) is low, it is also based on providers already having the tools needed to create the cards, such as a printer, laminator, and lamination supplies. The staff time needed to conduct the interview and make the card accounts for the bulk of the intervention's cost. We viewed this as an important investment due to the ability to build relationships between residents and staff through the process. However, we also recognized that some providers were able to use volunteers to implement the intervention. Therefore, we encouraged providers to delegate aspects of the PAL Card process when possible to contain costs and sustain the intervention. In addition, we collected the time needed for the initial learning curve. It is likely that costs may decrease as the learning curve levels off and staff become more efficient with PAL Card creation. It is unlikely that interview time would decrease substantially.

\section{Maintenance}


PAL Cards overwhelmingly remained in place throughout the duration of the project. Despite champions' concerns that cards would be accidentally removed or lost, only a small percentage of cards went missing or needed to be replaced. One consideration to maintaining the cards is that because they often are placed on a wheelchair or walker, they are at risk of being lost when these ambulatory devices are cleaned. In addition, one provider discussed how residents occasionally ended up using wheelchairs/walkers that were not their own, which caused confusion when staff would associate the PAL Cards with the wrong individual. While the majority of PAL Cards remained in place, it is unknown to what extent providers continued on with the project. Prior research on evidence-based programs has found that long-term implementation suffers once intervention supports (such as an external research team) are removed [33]. Staff turnover is another potential barrier to maintenance if the responsibilities for interviewing and PAL Card creation are not incorporated into staff job descriptions and standard operating procedures.

\section{Limitations and Future Directions:}

The findings of this study are limited by several factors. First, providers self-selected into the QIP. It is possible that the characteristics of the providers who opted in are different compared to providers who either chose another QIP offered by the State, or did not need a QIP during the timeframe ours was offered. In addition, we were only able to collect data from providers who completed the project. Despite multiple follow up email and telephone calls, we were unable to reach some of the participants to followup. Therefore, we have very limited information on those providers who did not complete. Learning more about those who did not complete the project would help us better understand the barriers to implementation. Additionaly, all the data in this study was self-reported. The findings, such as time to complete PELI interviews and PAL Cards, may not accurately represent the actual time as providers may have 'rounded up' or estimated the length of time either the interview or PAL Card creation took. In addition, the cost of implementing the PAL Cards should also be interpreted with caution. Staff wages used to calculate the costs were taken from online sources, which may not accurately reflect the wages of providers in Ohio. Additionally, there may be a wider range of costs than what was presented depending on the specific staff position. For example, within the category of nursing, there are a variety of positions including LPNs, RNs, and DONs, which have a wide range of salaries. We did not ask for staff to specify beyond 'nursing' as a department. We estimated the value of volunteers because while volunteers may not encumber salary costs, there are costs associated with their training and supervision. We recommend that providers using volunteers be trained in how to conduct the PELI interviews (training video available at www.preferencebasedliving.com at no cost) [34]. In the future, qualitative research on PAL Card implementation is needed to understand the varying approaches providers used. The results of this study show a large variation in the interview time and PAL Card creation time, which makes it difficult to generalize to what other providers will experience. Obtaining qualitative data on the barriers and facilitators to implementing the PAL Cards will allow for the implementation process to be improved.

\section{Conclusion}


There is a need to evaluate the implementation of evidence-based quality improvement projects in nursing homes. Quality improvement programs in healthcare are mostly studied in hospital settings, with very few studies in nursing homes [30]. This study helped fill this gap by evaluating the implementation of a quality improvement program among 26 nursing home providers. An external, virtual, QI support team and opportunities for learning help providers problem solve and overcome barriers. Building a flexible quality improvement program that was supported by an external research team facilitated implementation for a majority of providers who successfully completed the project. This study helped providers build the capacity to engage in person-centered care through creating PAL Cards for residents. Providers perceived the cards to be highly acceptable, feasible, and appropriate, which supports that the PAL Cards are viewed positively by providers and can be a useful tool for sharing preference information. Compared to other care settings, nursing homes are often resource-constrained and have high staff turnover [13], so a key feature of the PAL Cards are that they are relatively inexpensive, flexible to staff time limitations, and adds to the capacity of organizations to deliver valued person-centered care. In addition, these findings can be applied to other quality improvement interventions in residential settings, such as Assisted Living. The findings of this study aid in adapting implementation process for future projects.

\section{Abbreviations}

QI= quality improvement

QIP = quality improvement project

QAPI = Quality Assurance Performance Improvement

PAL Card $=$ Preference for Activity and Leisure

PELI-NH = Preferences for Everyday Living Inventory - Nursing Home Version

CMS $=$ Centers for Medicare and Medicaid Services

MDS = Minimum Data Set

ODA = Ohio Department of Aging

AIM = Acceptability of Intervention

IAM = Intervention Appropriateness Measure

FIM = Feasibility of Intervention Measure

RE-AIM = Reach, Efficacy, Adoption, Implementation, and Maintenance

PCC $=$ Person-Centered Care

Page $14 / 19$ 
LTSS $=$ long-term services and supports

$\mathrm{NH}=$ Nursing Home

\section{Declarations}

\section{Ethics approval and consent to participate}

The Miami University IRB Approved this study (protocol \#02686r The Acceptability, Appropriateness, and Feasibility of implementing PAL Cards in the Nursing Home setting. Verbal consent to participate was received by the provider participants.

\section{Consent for publication}

$\mathrm{N} / \mathrm{A}$

\section{Availability of data and materials}

The deidentified datasets used and analyzed for the current study are available by request from the corresponding author.

\section{Competing interests}

The authors declare that they have no competing interests.

\section{Funding}

Funding for this project was made possible through the Ohio Department of Medicaid.

\section{Authors' contributions}

All authors have approved of the submitted manuscript version and agree both to be personally accountable for their contributions and ensure that questions will be resolved.

a. Dr. Abbott conceptualized the study, designed the study, assisted with data collection, analysis, and drafted the manuscript.

b. Ms. Heppner assisted with data collection, analysis, and assisted with writing the manuscript

c. Dr. Hicks assisted with writing up methods and results and a critical final review of the final paper

d. Ms. Hermesch assisted with analysis and writing of the manuscript

e. Dr. VanHatisma assisted with the conceptualization and design of the study and drafting, editing, and manuscript revisions.

\section{Acknowledgements}


The authors wish to thank the nursing home providers who participated in the project as well as Morgan Liddic, a graduate student who assisted with data collection.

\section{References}

1. Koren MJ. Person-centered care for nursing home residents: the culture-change movement. Health Aff. 2010;29(2):312-7.

2. American Geriatrics Society Expert Panel on Person-Centered Care, Brummel-Smith K, Butler D, Frieder M, Gibbs N, Henry M, Koons E, Loggers E, Porock D, Reuben DB, Saliba D. Person-centered care: a definition and essential elements. J Am Geriatr Soc. 2016;64(1):15-8.

3. Saliba D, Buchanan J. Development and validation of a revised nursing home assessment tool: MDS 3.0. Santa Monica, CA: Rand Corp. 2008.

4. Van Haitsma KS, Curyto K, Abbott KM, Towsley GL, Spector A, Kleban M. A randomized controlled trial for an individualized positive psychosocial intervention for the affective and behavioral symptoms of dementia in nursing home residents. J Gerontol B Psychol Sci Soc Sci. 2015;70(1):3545 .

5. Kolanowski A, Litaker M, Buettner L, Moeller J, Costa, Jr PT. A randomized clinical trial of theorybased activities for the behavioral symptoms of dementia in nursing home residents. J Am Geriatr Soc. 2011;59(6):1032-41.

6. de Oliveira AM, Radanovic M, Homem de Mello PC, Buchain PC, Dias Vizzotto A, Harder J, Stella F, Piersol CV, Gitlin LN, Forlenza OV. An intervention to reduce neuropsychiatric symptoms and caregiver burden in dementia: preliminary results from a randomized trial of the tailored activity program-outpatient version. Int J Geriatr Psychiatry. 2019;34(9):1301-7.

7. Elliot, A. E., \& Barsness, S. (2016). What's in your bundle? Building a Communication Map of Relational Coordination Practices to Engage Staff in Individualizing Care. Seniors Housing \& Care Journal, 24(1), 1-19.

8. Tan M, Evans $\mathrm{KH}$, Braddock $\mathrm{CH}$, Shieh L. Patient whiteboards to improve patient-centred care in the hospital. Postgrad Med J. 2013;89(1056):604-9.

9. Jangland E, Carlsson M, Lundgren E, Gunningberg L. The impact of an intervention to improve patient participation in a surgical care unit: a quasi-experimental study. Int J Nurs Stud. 2012;49(5):528-38.

10. Murphy J, Gray CM, van Achterberg T, Wyke S, Cox S. The effectiveness of the talking mats framework in helping people with dementia to express their views on well-being. Dementia. 2010;9(4):454-72.

11. Dijkstra K, Bourgeois M, Burgio LO, Allen R. Effects of a communication intervention on the discourse of nursing home residents with dementia and their nursing assistants. J Med Speech Lang Pathol. 2002;10(2):143-58. 
12. Moos I, Björn A. Use of the life story in the institutional care of people with dementia: a review of intervention studies. Ageing Soc. 2006;26(3):431-54.

13. Ginsburg L, Easterbrook A, Berta W, Norton P, Doupe M, Knopp-Sihota J, Anderson RA, Wagg A. Implementing frontline worker-led quality improvement in nursing homes: getting to "how". Jt Comm J Qual Patient Saf. 2018;44(9):526-35.

14. Eccles MP, Mittman BS. Welcome to implementation science. Implementation Science. 2006;1:1.

15. Baier RR, Jutkowitz E, Mitchell SL, McCreedy E, Mor V. Readiness assessment for pragmatic trials (RAPT): a model to assess the readiness of an intervention for testing in a pragmatic trial. BMC Med Res Methodol. 2019;19(1):156.

16. Saliba D, Buchanan J. Development and validation of a revised nursing home assessment tool: MDS 3.0. RAND Health Corporation. 2008.

17. Van Haitsma K, Curyto K, Spector A, Towsley G, Kleban M, Carpenter B, Ruckdeschel K, Feldman PH, Koren MJ. The preferences for everyday living inventory: Scale development and description of psychosocial preferences responses in community-dwelling elders. Gerontologist. 2013;53(4):582-95.

18. Heid AR, Eshraghi K, Duntzee $\mathrm{Cl}$, Abbott K, Curyto K, Van Haitsma K. "It depends": Reasons why nursing home residents change their minds about care preferences. Gerontologist. 2016;56(2):24355 .

19. Van Haitsma K, Abbott KM, Heid AR, Carpenter B, Curyto K, Kleban M, Eshraghi K, Duntzee Cl, Spector A. The consistency of self-reported preferences for everyday living: Implications for person-centered care delivery. J Gerontol Nurs. 2014;40(10):34-46.

20. Ohio Administrative Code. Long-term care quality initiative. http://codes.ohio.gov/oac/173-60. Accessed 7 Sept 2020.

21. Glasgow RE, Vogt TM, Boles SM. Evaluating the public health impact of health promotion interventions: the RE-AIM framework. Am J Public Health. 1999 Sep;89(9):1322-7. doi: 10.2105/ajph.89.9.1322. PMID: 10474547; PMCID: PMC1508772.

22. Pinnock H, Barwick M, Carpenter C, Eldridge S, Grandes G, Griffiths CJ, Rycroft-Malone J, Meissner P, Murray E, Patel A, Sheikh A, Taylor SJC for the StaRI Group. Standards for Reporting Implementation Studies (StaRI) statement. BMJ 2017;356:i6795

23. Ohio Department of Aging. Nursing home quality improvement. https://aging.ohio.gov/wps/portal/gov/aging/agencies-and-service-providers/nursing-home-qualityimprovement/. Accessed 7 Sept 2020.

24. Institute for Healthcare Improvement. How to improve. http://www.ihi.org/resources/Pages/Howtolmprove/default.aspx. Accessed 7 Sept 2020.

25. Action Pact. The learning circle. http://actionpact.com/assets/cache/learning-circle.pdf. Accessed 7 Sept 2020.

26. Keane B. The learning circle in culture change: Why use it? https://www.pioneernetwork.net/wpcontent/uploads/2016/10/The-Learning-Circle-in-Culture-Change.pdf. Accessed 7 Sept 2020. 
27. Weiner BJ, Lewis CC, Stanick C, Powell BJ, Dorsey CN, Clary AS, Boynton MH, Halko H. Psychometric assessment of three newly developed implementation outcome measures. Implement Sci. 2017;12(1):1-2.

28. The Value of Volunteer Time/State and Historical Data Dataset. https://independentsector.org/resource/vovt_details/. Accessed 7 Sept 2020.

29. Rantz MJ, Zwygart-Stauffacher M, Flesner M, Hicks L, Mehr D, Russell T, Minner D. Challenges of using quality improvement methods in nursing homes that "need improvement". J Am Med Dir Assoc. 2012;13(8):732-8.

30. Woo K, Milworm G, Dowding D. Characteristics of quality improvement champions in nursing homes: a systematic review with implications for evidence-based practice. Worldviews Evid Based Nurs. 2017;14(6):440-6.

31. Proctor E, Silmere H, Raghavan R, Hovmand P, Aarons G, Bunger A, Griffey R, Hensley M. Outcomes for implementation research: conceptual distinctions, measurement challenges, and research agenda. Adm Policy Ment Health. 2011;38(2):65-76.

32. Abbott KM, Heid AR, Kleban M, Rovine MJ, Van Haitsma K. The Change in Nursing Home Residents' Preferences Over Time. J Am Med Dir Assoc. 2018;19(12):1092-8.

33. Berta WB, Wagg A, Cranley L, Doupe MB, Ginsburg L, Hoben M, MacEachern L, Chamberlain S, Clement F, Easterbrook A, Keefe JM. Sustainment, sustainability, and spread study (SSaSSy): protocol for a study of factors that contribute to the sustainment, sustainability, and spread of practice changes introduced through an evidence-based quality-improvement intervention in Canadian nursing homes. Implement Sci. 2019;14(1):109.

34. Preference Based Living. https://www.preferencebasedliving.com/. Accessed 7 Sept 2020.

\section{Figures}
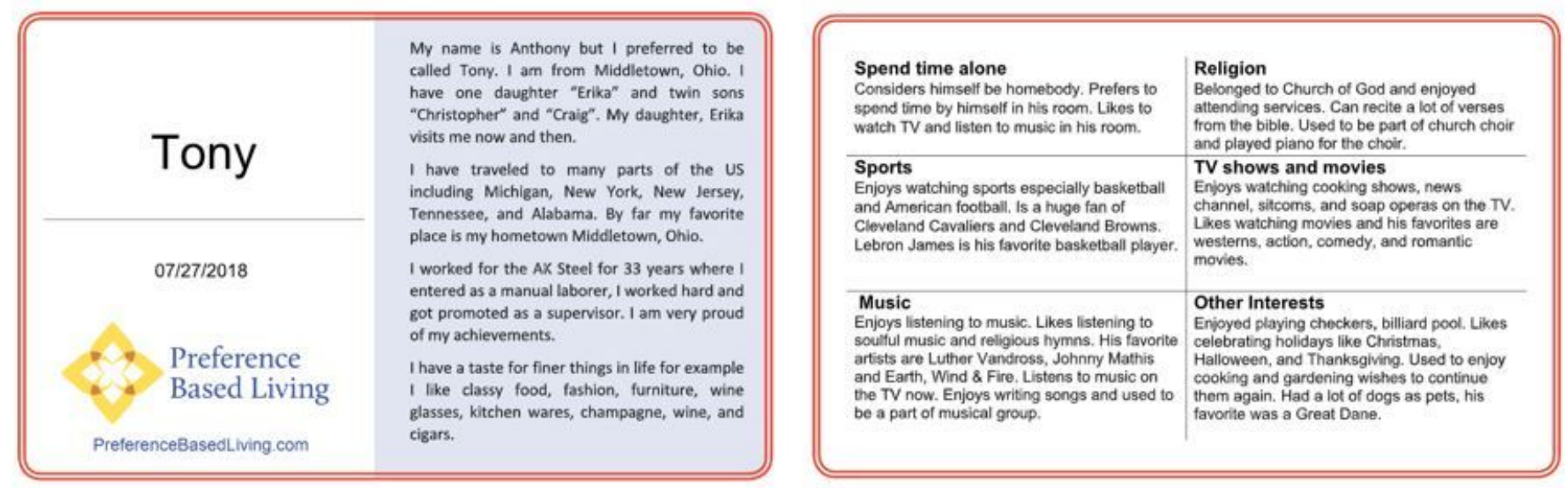

Figure 1 


\begin{tabular}{|c|c|c|c|c|c|c|c|c|}
\hline $\begin{array}{c}40 \text { Providers } \\
\text { Registered for the } \\
\text { QIP }\end{array}$ & $\begin{array}{c}\text { Attended 1 hour } \\
\text { Virtual Training }\end{array}$ \\
35 Providers Began \\
Implementation
\end{tabular}

Figure 2

Consort Diagram of Provider Participation and PAL Card Creation

\section{Supplementary Files}

This is a list of supplementary files associated with this preprint. Click to download.

- StaRIchecklistAbbottPCCommunicationTool.docx 